\title{
Pion flow and antiflow in relativistic heavy-ion collisions
}

\author{
Bao-An Li and C. M. Ko \\ Cyclotron Institute and Physics Department, Texas A\&M University, College Station, Texas 77843
}

(Received 13 July 1995)

\begin{abstract}
Within the framework of a relativistic transport model (ART 1.0) for heavy-ion collisions at AGS energies we study the transverse flow of pions with respect to that of nucleons using two complementary approaches. It is found that in central collisions pions develop a weak flow as a result of the flow of baryon resonances from which they are produced. On the other hand, they have a weak antiflow in peripheral collisions due to the shadowing of spectators. Furthermore, it is shown that both pion flow and antiflow are dominated by those with large transverse momenta.
\end{abstract}

PACS number(s): 25.75.Ld, 24.10.Jv, 24.10.Nz

A major highlight of recent experiments at Brookhaven's AGS is the discovery of sideward collective flow of nucleons in reactions of $\mathrm{Au}+\mathrm{Au}$ at $p_{\text {beam }} / A=10.8 \mathrm{GeV} / c$ [1,2]. In view of the large number of pions created in these reactions and the strong coupling between the nucleon and pion, it is interesting to know if pions also have a collective flow behavior and, if yes, how the pion flow is related to the nucleon flow. These questions have recently been addressed in experiments by the E877 Collaboration by studying the correlation between pion transverse momentum distributions in the reaction plane and the direction of nucleon collective flow near the projectile rapidity. In these experiments, the strength and direction of pion collective flow were measured by examining the multiplicity ratio $R$ of pions emitted in the direction of nucleon flow over those emitted in the opposite direction of nucleon flow. The pion flow or antiflow is then characterized quantitatively by the ratio $R$ being larger or smaller than one. Surprisingly, from the $13 \%$ most central collisions where a large strength of nucleon flow was found [3], preliminary data with relatively large errors show neither flow nor antiflow signatures for pions. On the other hand, theoretical calculations using RQMD [4] and ARC [5] have predicted the existence of pion antiflow for the minimum biased events in $\mathrm{Au}+\mathrm{Au}$ reactions. However, these calculations do not provide the much needed information about the impact parameter dependence of the pion flow or antiflow in order to understand the experimental results. In this paper we carry out a detailed study on the pion flow and antiflow for both central and peripheral collisions using two complementary approaches. We find that pions show a weak flow behavior in central collisions due to the flow of baryon resonances from which they are produced but a weak antiflow behavior in peripheral collisions as a result of the shadowing of spectators. Moreover, it is shown that both pion flow and antiflow are dominated by those with large transverse momenta.

Our study is based on the relativistic transport model (ART 1.0) developed recently for heavyion collisions at AGS energies [6]. In this model the phase space distribution functions of baryons $\left[N, \Delta(1232), N^{*}(1440), N^{*}(1535), \Lambda, \Sigma\right]$ and mesons $(\pi, \rho, \omega, \eta, K)$ are evolved under the influence of hadronhadron scatterings and also an optional mean field for baryons. The model has been rather successful in studying many aspects of heavy-ion collisions at AGS energies. We refer the reader to our previous work for more details [6].

In order to identify signatures of pion flow or antiflow we use two complementary approaches. One is based on the analysis of the average transverse momentum in the reaction plane as a function of rapidity [7], and the other is the analysis of the strength of flow as a function of transverse momentum at a constant rapidity [3,8]. First, we show in Fig. 1 the average transverse momentum of nucleons and pions in the reaction plane as a function of rapidity for both central (with impact parameters of $b \leqslant 4 \mathrm{fm}$ ) and peripheral (with impact parameters of $8 \mathrm{fm} \leqslant b \leqslant 10 \mathrm{fm}$ ) collisions. It is seen that the average transverse momentum of pions is much smaller than that of nucleons. This seems in agreement with the experimental observations. However, more detailed study reveals that pions near target or projectile rapidities have a weak flow behavior in central collisions and an antiflow behavior in peripheral collisions with respect to the flow direction of nucleons. The reason that pions flow (antiflow) in central (peripheral) collisions is due to the fact that the direction of

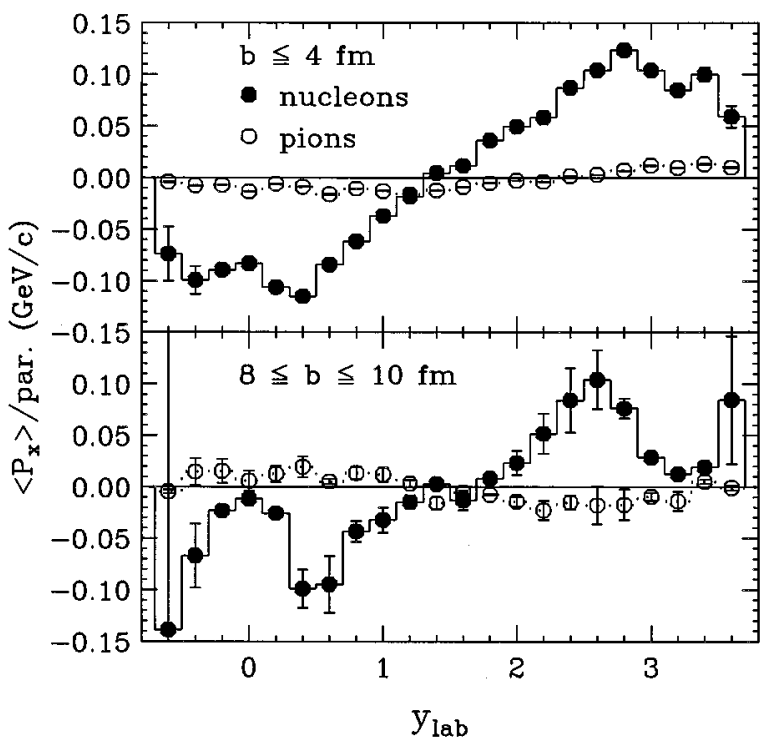

FIG. 1. The average transverse momentum of nucleons and pions in the central (upper window) and peripheral (lower window) collisions of $\mathrm{Au}+\mathrm{Au}$ at $p_{\text {beam }} / A=10.8 \mathrm{Gev} / \mathrm{c}$. 


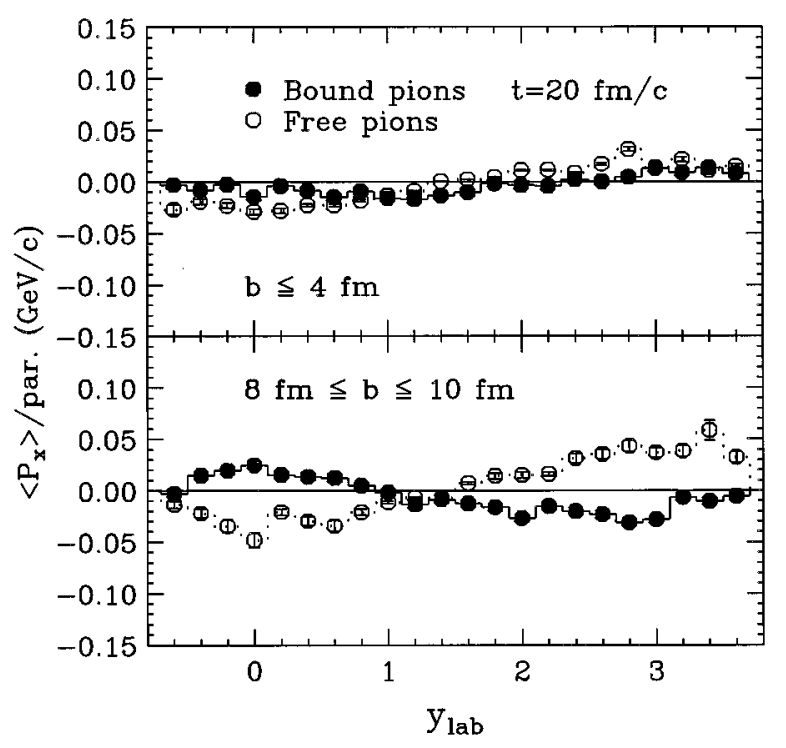

FIG. 2. The average transverse momentum of bound and free pions in the central (upper window) and peripheral (lower window) collisions of $\mathrm{Au}+\mathrm{Au}$ at $p_{\text {beam }} / A=10.8 \mathrm{Gev} / c$.

pion flow (or the sign of average transverse momenta) is a result of the competition between the collective flow of baryon resonances and the shadowing of spectators through rescatterings and reabsorptions. In our model pions are produced either directly from particle-particle collisions or from the decay of resonances. In central collisions pions are produced throughout the whole reaction volume, and there is thus little shadowing effect. If the colliding particle pairs or baryon resonances which produce pions have a large flow velocity, then the produced pions would also have a certain flow velocity in the same direction as the nucleon as a result of momentum conservation. However, because of the production kinematics, the pion flow is much reduced. On the other hand, in peripheral collisions the shadowing effect from the spectators dominates and therefore results in the apparent antiflow of pions in the opposite direction of nucleon flow. We find that the transition from pion flow to antiflow occurs at an impact parameter of about $7 \mathrm{fm}$ for the $\mathrm{Au}+\mathrm{Au}$ reaction at $p_{\text {beam }} / A=10.8 \mathrm{GeV} / c$. It is interesting to mention that the transition from pion flow to antiflow at an impact parameter of about $3 \mathrm{fm}$ has long been predicted at BEVALAC and/or SIS/GSI energies by several groups [9-12] and has been confirmed recently by experiments $[13,14]$. The smaller shadowing effects at AGS energies as indicated by the smaller pion transverse momentum and a larger transition impact parameter at which it becomes important is due to the fact that the spectators fly away with very large longitudinal momenta in these reactions and thus have less effects on pions from the participant region as compared to heavy ion collisions at SIS/GSI energies.

The above explanation is more clearly demonstrated in Fig. 2 where contributions to the average transverse momentum from pions already existing (free pions) and pions from the decay of baryon resonances which are still present at 20 $\mathrm{fm} / c$ (bound pions) are shown separately. We would like to stress that the relative multiplicities of the two kinds of pions change gradually during the reaction as shown in Fig. 3 for

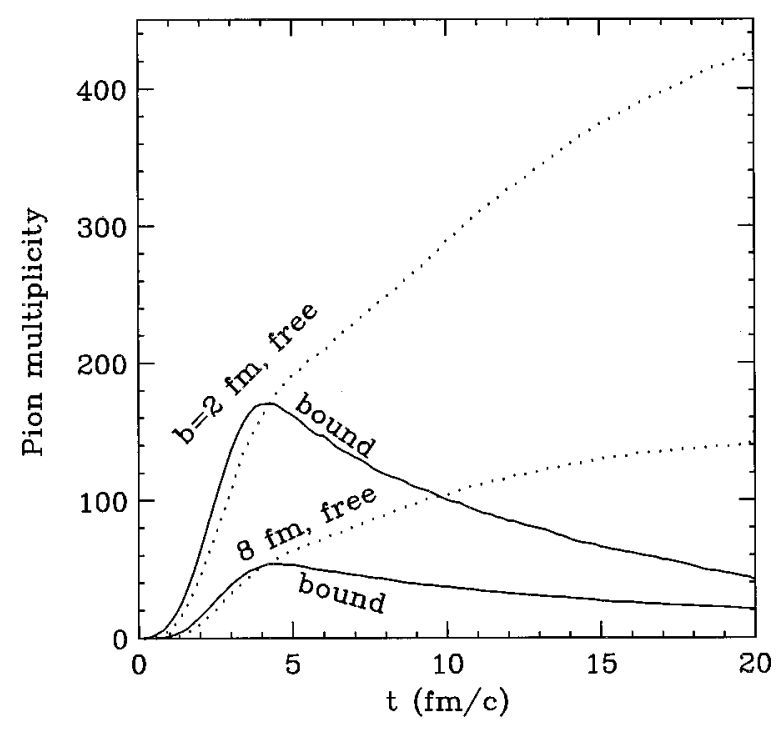

FIG. 3. The evolution of pion multiplicities in the reactions of $\mathrm{Au}+\mathrm{Au}$ at $p_{\text {beam }} / A=10.8 \mathrm{GeV} / c$ and impact parameters of $2 \mathrm{fm}$ and $8 \mathrm{fm}$.

the reaction of $\mathrm{Au}+\mathrm{Au}$ at $p_{\text {beam }} / A=10.8 \mathrm{GeV} / c$ and impact parameters of $2 \mathrm{fm}$ and $8 \mathrm{fm}$. Because of the large pionnucleon cross section, pions freeze-out rather late. At $t=20$ $\mathrm{fm} / c$, the multiplicity ratio of bound/free pions is about $40 /$ 420 and 20/140 at impact parameters of $2 \mathrm{fm}$ and $8 \mathrm{fm}$, respectively. From Fig. 2 it is seen that bound pions have a typical $S$-shaped transverse momentum distribution similar to those for nucleons in both central and peripheral collisions. The apparent flow behavior of bound pions is due to the collective flow of baryon resonances from which they are produced. However, free pions produced earlier either directly from particle-particle collisions or from decays of resonances have generally gone through several annihilationproduction cycles which can destroy their collectivity, and more importantly they also have more chance to be rescattered by the spectators. In central collisions free pions show less collectivity than bound pions, but still flow in the same direction as nucleons. In peripheral collisions, however, these pions show a distinct antiflow behavior due to the shadowing of spectators. The final pion transverse momentum distribution therefore reflects the complicated reaction dynamics of pion production, reabsorption, rescattering as well as the collective flow of baryon resonances. The study of pion flow or antiflow may therefore reveal interesting information about the in-medium cross sections of elementary processes involving pions and baryon resonances.

Since the currently available spectrometers at the AGS do not allow a complete analysis of the average transverse momentum as a function of rapidity, a complementary approach, i.e., the analysis of the pion transverse momentum spectrum in the reaction plane at a fixed rapidity, has been used [3]. In this approach, the ratio $R\left(p_{t}\right) \equiv\left(d N^{+} / d p_{t}\right) /\left(d N^{-} / d p_{t}\right)$, where $N^{+}\left(N^{-}\right)$is the number of particles emitted in the reaction plane in the same (opposite) direction of nucleon sideward flow, is examined as a function of transverse momentum near the projectile rapidity. To increase the statistics of our analysis particles with azimuthal angles smaller than $20^{\circ}$ with respect to the reaction plane and within the rapidity 


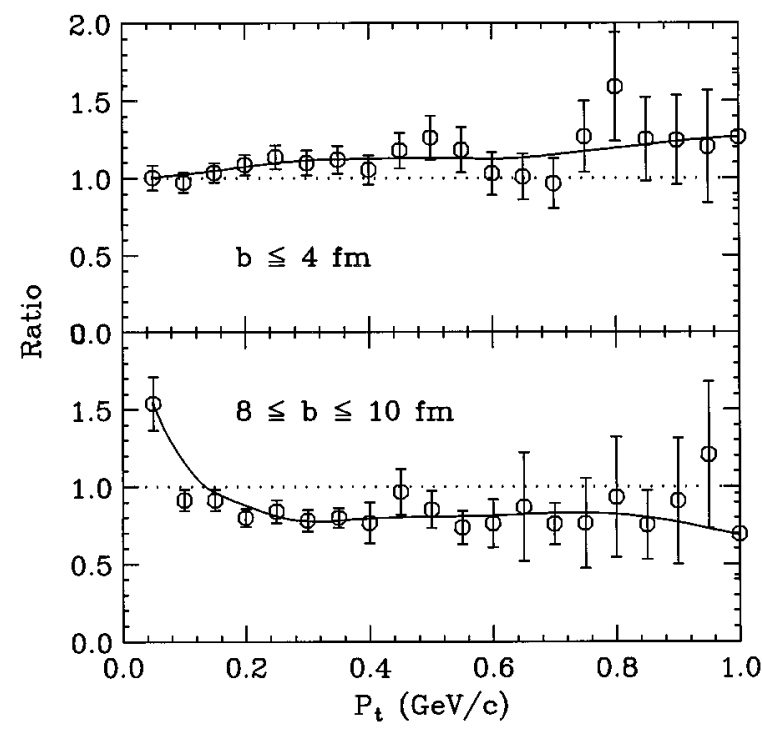

FIG. 4. The transverse momentum dependence of the strength $R\left(p_{t}\right)$ of transverse flow in the reactions of $\mathrm{Au}+\mathrm{Au}$ at $p_{\text {beam }} / A=10.8 \mathrm{GeV} / c$ and impact parameters of $2 \mathrm{fm}$ and $8 \mathrm{fm}$.

range of $|y-2.95| \leqslant 0.35$ are included. We note that the projectile rapidity of 3.1 for the $\mathrm{Au}+\mathrm{Au}$ reaction at $p_{\text {beam }} / A=10.8 \mathrm{GeV} / c$ is in this rapidity bin. It is clear that the observation of $R\left(p_{t}\right)>1$ at all transverse momenta is an unambiguous signature of the existence of sideward flow, and its variation with the transverse momentum can also give a detailed measure of the strength of collective flow. This approach was recently found to provide new information about the collective flow that is complementary to that obtained from the analysis of the average transverse momentum in the reaction plane [8].

Figure 4 shows the ratio $R\left(p_{t}\right)$ as a function of $p_{t}$ for pions from the central and peripheral collisions of the $\mathrm{Au}+\mathrm{Au}$ reaction at $p_{\text {beam }} / A=10.8 \mathrm{GeV} / c$. The solid lines are drawn to guide the eyes. In central collisions, there is a clear tendency of pion flow in the same direction as nucleons if their transverse momenta are larger than $0.2 \mathrm{GeV} / c$. For large transverse momentum pions in peripheral collisions, we have $R\left(p_{t}\right) \leqslant 1$ indicating that there is a tendency of antiflow. It is also interesting to note that very low transverse momentum pions with $p_{t} \leqslant 0.1 \mathrm{GeV} / c$ in peripheral collisions are preferentially emitted in the direction of nucleon flow. From the lower window of Fig. 2 we see that these pions are mainly from the decay of baryon resonances after the freezeout. These resonances generally have light masses and similar transverse momentum distributions as nucleons. The decay of these resonances therefore emits preferentially low transverse momentum pions along the direction of nucleon flow. Although the impact parameters of central collisions studied here are close to those of the $13 \%$ most central $\mathrm{Au}+\mathrm{Au}$ collisions studied by the E877 Collaboration, a direct comparison of our results with the experimental data requires a careful simulation of the experimental filters. Nevertheless, our study indicates that one needs to be cautious in searching for pion antiflow in central heavy ion collisions.

In summary, we have studied the transverse flow of pions with respect to that of nucleons using two complementary approaches. Within the framework of the relativistic transport model (ART 1.0) for heavy-ion collisions at AGS energies, pions are found to have a weak flow behavior due to the flow of baryon resonances in central collisions. In peripheral collisions pions have instead a weak antiflow behavior as a result of the shadowing of spectators. Furthermore, it is found that both pion flow and antiflow are dominated by those with large transverse momenta. These results are expected to be useful for detailed experimental studies of pion flow and antiflow in relativistic heavy-ion collisions.

We would like to thank P. Danielewicz and G.Q. Li for helpful discussions. B.A.L. also acknowledges the kind hospitality extended to him by the nuclear theory group at Michigan State University where part of this work was carried out. This work was supported in part by NSF Grant No. PHY-9212209 and the Welch Foundation Grant No. A-1110. The support of C.M.K. by a Humboldt Research Award is also gratefully acknowledged, and he would like to thank Ulrich Mosel of the University of Giessen for the warm hospitality.
[1] E877 Collaboration, J. Barrette et al., Phys. Rev. Lett. 73, 2532 (1994).

[2] E877 Collaboration, Yingchao Zhang and J.P. Wessels, in Proceedings of Quark Matter '95, Monterey, CA, Jan. 9-13, 1995 [Nucl. Phys. A590, 557c (1995)].

[3] E877 Collaboration, J. Barrette et al., in Proceedings of Quark Matter '95 [2] [Nucl. Phys. A590, 259c (1995)].

[4] A. Jahns, C. Spieles, H. Sorge, H. Stöcker, and W. Greiner, Phys. Rev. Lett. 72, 3464 (1995).

[5] D.E. Kahana, D. Keane, Y. Pang, T. Schlagel, and S. Wang, Phys. Rev. Lett. 74, 4406 (1995).

[6] B.A. Li and C.M. Ko, Phys. Rev. C 52, 2037 (1995).

[7] P. Danielewicz and G. Odyniec, Phys. Lett. 157B, 146 (1985).
[8] B.A. Li, C.M. Ko, and G.Q. Li, Phys. Lett. B (to be published).

[9] B.A. Li, W. Bauer, and G.F. Bertsch, Phys. Rev. C 44, 2095 (1991).

[10] S.A. Bass, R. Mattiello, H. Stöcker, W. Greiner, and Ch. Hartnack, Phys. Lett. B 302, 381 (1993).

[11] B.A. Li, Phys. Lett. B 319, 412 (1993); Nucl. Phys. A570, 797 (1994).

[12] P. Danielewicz, Phys. Rev. C 51, 716 (1995).

[13] FOPI Collaboration, D. Pelte et al., Nachrichten GSI 09-93, p. 4, 1993.

[14] EOS Collaboration, J.C. Kintner et al., Phys. Rev. Lett. (submitted). 